\title{
Minimal ablative margin (MAM) assessment with image fusion: an independent predictor for local tumor progression in hepatocellular carcinoma after stereotactic radiofrequency ablation
}

\author{
Gregor Laimer ${ }^{1} \cdot$ Peter Schullian $^{1} \cdot$ Nikolai Jaschke $^{2} \cdot$ Daniel Putzer $^{1} \cdot$ Gernot Eberle $^{1} \cdot$ Amilcar Alzaga $^{3}$. \\ Bruno Odisio $^{4} \cdot$ Reto Bale $^{1}$ (D)
}

Received: 1 November 2019 / Revised: 22 November 2019 / Accepted: 5 December 2019 / Published online: 30 January 2020

(C) The Author(s) 2019

\begin{abstract}
Objectives To assess the minimal ablative margin (MAM) by image fusion of intraprocedural pre- and post-ablation contrastenhanced CT images and to evaluate if it can predict local tumor progression (LTP) independently. Furthermore, to determine a MAM with which a stereotactic radiofrequency ablation (SRFA) can be determined successful and therefore used as an intraprocedural tool to evaluate treatment success.

Methods A total of 110 patients (20 women, 90 men; mean age $63.7 \pm 10.2$ ) with 176 hepatocellular carcinomas were assessed by retrospective analysis of prospectively collected data. The MAM was determined through image fusion of intraprocedural preand post-ablation images using commercially available rigid imaging registration software. LTP was assessed in contrastenhanced CTs or MR scans at 3-6-month intervals.

Results The MAM was the only significant independent predictor of LTP $(p=0.036)$. For each millimeter increase of the MAM, a $30 \%$ reduction of the relative risk for LTP was found (OR $=0.7,95 \%$ CI $0.5-0.98, p=0.036)$. No LTP was detected in lesions with a MAM $>5 \mathrm{~mm}$. The overall LTP rate was 9 of $110(8.2 \%)$ on a patient level and 10 of $173(5.7 \%)$ on a lesion level. The median MAM was 3.4 (1.7-6.9) mm. The mean overall follow-up period was $26.0 \pm 10.3$ months.

Conclusions An immediate assessment of the minimal ablative margin (MAM) can be used as an intraprocedural tool to evaluate the treatment success in patients treated with stereotactic RFA. A MAM $>5 \mathrm{~mm}$ has to be achieved to consider an ablation as successful.

Key Points

- An intraoperatively measured minimal ablative margin (MAM) > $5 \mathrm{~mm}$ correlates with complete remission.

- MAM is the only significant independent predictor of LTP (OR=0.7, 95\% CI 0.5-0.98, p=0.036) after stereotactic RFA of hepatocellular carcinoma.

- Image fusion using commercially available rigid imaging registration software is possible, even though considerably complex. Therefore, improved (semi-)automatic fusion software is highly desirable.
\end{abstract}

Keywords Ablation techniques · Radiofrequency ablation · Carcinoma, hepatocellular · Tomography, X-ray computed · Treatment outcome

Reto Bale

reto.bale@i-med.ac.at

1 Department of Radiology, Interventional Oncology-Microinvasive Therapy (SIP), Medical University Innsbruck, Anichstr. 35, 6020 Innsbruck, Austria
2 Department of Internal Medicine I, Gastroenterology, Hepatology, Endocrinology and Metabolism, Medical University Innsbruck, Anichstr. 35, 6020 Innsbruck, Austria

3 Siemens Healthineers, Siemensstraße 3, 91301 Forchheim, Germany

4 Division of Diagnostic Imaging, Department of Interventional Radiology, The University of Texas MD Anderson Cancer Center, Houston, TX, USA 


$\begin{array}{ll}\text { Abbreviations } \\ \text { AASLD } & \begin{array}{l}\text { American Association for the Study of Liver } \\ \text { Diseases }\end{array} \\ \text { CT } & \text { Computed tomography } \\ \text { EASL } & \text { European Association for the Study of the Liver } \\ \text { HCC } & \text { Hepatocellular carcinoma } \\ \text { LTP } & \text { Local tumor progression } \\ \text { MAM } & \text { Minimal ablative margin } \\ \text { RFA } & \text { Radiofrequency ablation } \\ \text { SBRT } & \text { Stereotactic body radiotherapy } \\ \text { SRFA } & \text { Stereotactic radiofrequency ablation }\end{array}$

Introduction

In recent years, radiofrequency ablation (RFA) has emerged as a first-line curative treatment approach for patients with small hepatocellular carcinoma (HCC) lesions [1-4]. The 2018 American Association for the Study of Liver Diseases (AASLD) guideline considers RFA and surgical resection as equal treatment options for single tumors $<2.5 \mathrm{~cm}$ [5]. Major complications after RFA are rare and treatment-associated mortality is extremely low [6-11].

Technical limitations of the conventional computed tomography (CT) - or US-guided techniques can be overcome by multiple-needle approaches using 3D treatment planning and stereotactic needle placement (termed "stereotactic RFA" or "SRFA") [12-20].

A crucial point in curative treatments of $\mathrm{HCC}$ is the evaluation of the treatment success. In patients treated with surgical resection, the histopathological examination of the surgical specimen gives certainty whether the resection margin is free of cancer cells (R0), cancer cells are present microscopically (R1), or residual gross tumor tissue is present (R2). The resection margin has a significant influence on prognosis of patients with $\mathrm{HCC}$ [21-23], and it determines the treatment plan to follow. After radiation therapy, such as SBRT, an immediate assessment of treatment efficacy is not possible. The therapeutic effect can only be seen in subsequent follow-up imaging.

Up until now, it has been similar in RFA. The treatment efficacy of RFA could only be assessed through the absence of residual tumor on 1-month follow-up imaging or through the absence of local tumor progression (LTP) in subsequent follow-up imaging. An intraprocedural, more contemporaneous assessment of treatment success after RFA is highly desirable.

Accumulating evidence has pointed towards the postablation safety margin (minimal ablative margin) as a critical determinant of RFA success. In several studies, a minimal ablative margin (MAM) of less than $5 \mathrm{~mm}$ has been associated with higher rates of LTP [24-26]. Therefore, we postulate that the assessment of the MAM can be used as an intraprocedural tool to evaluate the treatment success of stereotactic RFA and facilitate further ablation in the same session if needed.

The aim of this study was to assess the minimal ablative margin (MAM) with image fusion of pre- and post-ablation CT images and to evaluate if it can predict LTP independently. Furthermore, we sought to determine a MAM with which a stereotactic RFA can be judged as successful.

\section{Material and methods}

\section{Patients}

This is an institutional review board approved, single-center, retrospective analysis of prospectively collected data from HCC patients referred to stereotactic RFA between January 2009 and February 2016.

In all patients, the treatment plan was established by a multidisciplinary tumor board consisting of hepatologists, oncologists, transplant surgeons, and interventional radiologists. Treatment choice was based on tumor characteristics, ChildPugh classification, anatomical considerations, and the general patient condition.

Inclusion criteria for stereotactic RFA were as follows: (1) Tumors showed the typical imaging characteristics with hypervascularity in the arterial phase and washout in the delayed portal venous phase on contrast-enhanced CT and were accessible by a percutaneous approach [27]; (2) prothrombin time ratio greater than $50 \%$ (prothrombin time with international normalized ratio, G1.7) and platelet count greater than 60,000 cells $/ \mathrm{mm}^{3} ;(3)$ absence of portal vein thrombosis and extrahepatic metastases.

Routine pre-operative evaluation of patients with hepatocellular carcinoma included baseline history, physical examination, and serum laboratory tests. Contrast-enhanced CT of the abdomen was performed for HCC diagnosis accordingly to the European Association for the Study of the Liver (EASL) [27]. In addition, chest CT was routinely performed to exclude the presence of pulmonary metastases. All HCCs included in this study were pathologically confirmed by needle biopsy during the stereotactic RFA procedure.

In total, 146 patients with 273 HCC lesions were identified. Exclusion criteria for retrospective evaluation of MAM were as follows: follow-up < 1 year; extensive liver deformation due to stereotactic RFA of large or multiple tumors leading to an inacceptable fusion result; multiple ablation sessions with incomplete ablation in a single session; multifocal diffuse tumor progression due to very aggressive tumor biology (Fig. 1). 
Fig. 1 Exclusion criteria for the evaluation of MAM using image fusion leading to $176 \mathrm{HCCs}$ in 110 patients

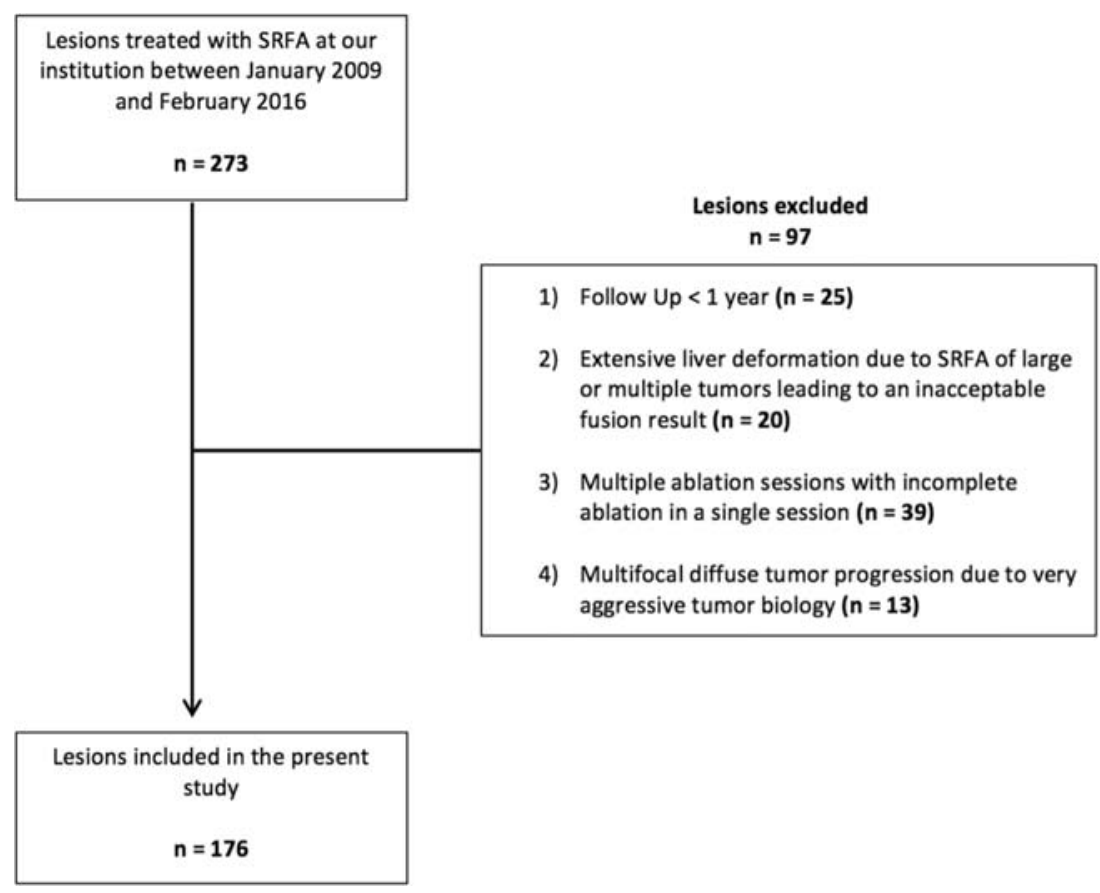

\section{Stereotactic radiofrequency ablation}

The detailed stereotactic RFA procedure has been described elsewhere $[12,13,19,20]$. Briefly, the procedure is performed in an interventional CT suite under general anesthesia and neuromuscular blockade. The patient is fixed on a CT table by a vacuum bag (Bluebag, Medical Intelligence). Ten to 15 registration markers for image to patient registration (Beekley Spots, Beekley Corporation) are attached to the skin of the thorax and the upper abdomen. Thereafter, a dual-phase contrast-enhanced planning CT (Siemens SOMATOM Sensation Open, sliding gantry with $82 \mathrm{~cm}$ diameter, Siemens AG) with $3-\mathrm{mm}$ slice thickness is acquired. Images are obtained $35-40$ and 70-80 s after initiation of contrast material injection (100$150 \mathrm{ml}$ of iopromide [Ultravist 370; Schering AG]), representing late arterial and late portal venous phases. All CT images are acquired with the patient in breath hold by temporary tracheal tube disconnection to overcome differences in position due to respiratory motion.

The CT data is then sent to the optical-based navigation system (Stealth Station Treon Plus, Medtronic Inc.). Multiple electrode positions are planned on the multiplanar and reconstructed images in order to cover the entire tumor volume. Following registration, accuracy check, and sterile draping, the ATLAS aiming device (Medical Intelligence) is manually adjusted using the guidance software of the stereotactic navigation system and $15 \mathrm{G}$ coaxial needles (Bard Inc.) are introduced through the aiming device to a pre-planned depth. A CT scan for verification of correct needle placement is obtained. A biopsy sample is taken via the coaxial needles using a 16-gauge core biopsy needle. Subsequently, three radiofrequency probes with a $3 \mathrm{~cm}$ active tip (Cool-tip, Medtronic) are introduced via the $15 \mathrm{G}$ coaxial needles, the latter being retracted to uncover the active probe exposure. At each position, ablations are performed using the switching control mode for up to three probes during the 16-min ablation per cycle. In case of significant increase of impedance (the so-called roll-off effect) the ablation process was terminated. After ablation completion and removal of RFA probes and co-axial needles by tract cauterization, a dual-phase contrast-enhanced CT scan is obtained for complication and ablation assessment.

A complete ablation was defined by a circumscribed nonenhancing area within and/or extending beyond tumor borders. Any areas of abnormally enhanced tissue in the late arterial phase and washout in the delayed phase that were located within or along the margin of the coagulation zone were considered residual tumor.

\section{Image fusion and evaluation of minimal ablative margin}

Computed tomography imaging fusion was performed using commercially available rigid imaging registration software (Syngo.via VB20A, Siemens Healthineers) with automatic registration followed by verification and, if required, manual registration. The arterial phase was utilized as the image of choice for pre-ablation images as the lesions demarcate clearly from the surrounding liver parenchyma which facilitated an accurate evaluation of the MAM. If possible, the late arterial phase was used as the post-ablation image dataset because the 
landmarks were clearly identifiable and could be easily correlated with the late arterial planning phase.

At first, images were registered automatically by a rigid registration tool included in the software. However, automatic registration was not satisfactory. Through manual correction by multiplanar slight shifting (translation and rotation) in axial, coronal, and sagittal planes referring to well defined intrahepatic structures, a satisfactory fusion was finally achieved. These so-called intrahepatic landmarks were in most cases vessel bifurcation of hepatic arteries or segmental branches of the portal vein. Landmarks close to the lesion, if possible in the same segment, were preferably used to fuse images accurately. In patients with more than one lesion, image registration was repeated for every lesion in other segments if necessary.

After successful fusion of pre- and post-ablation images, the distances between tumor border and margin of the necrosis zone in the axial, coronal, and sagittal planes were measured. The smallest distance was defined as the minimal ablative margin (MAM). The extension of the minimal ablative margin was described in hours using a 12-h scale of an analogue watch, as illustrated in Fig. 2. An example of successful image fusion and evaluation of MAM is shown in Figs. 3 and 4, while Fig. 5 shows an example of registration failure due to extensive liver deformation.

\section{Stereotactic RFA imaging response assessment and patient follow-up}

After stereotactic RFA, contrast-enhanced follow-up CTs with 3-mm slice thickness (including native, late arterial, late portal, and delayed phases) were performed at 3-6month intervals. For our study, we followed the treated tumors for a maximum time of 36 months for patients without local tumor progression (LTP). In patients with LTP, follow-up ended with the date of detection of the local tumor progression (i.e., event). In patients with liver transplantation after stereotactic RFA as bridging therapy, the histopathological exam of the explanted liver was used to determine LTP. Residual vital tissue was judged as LTP and follow-up ended with the date of liver transplantation (i.e., event).

Local tumor progression was defined as a newly detected nodular hypervascular lesion with washout in the late portal venous phase immediately adjacent to the ablation zone and detected within 36 months after the intervention (Fig. 3e). Newly detected tumors distant to the ablation zone were defined as distant tumor recurrence.

Both image fusion and evaluation of the minimal ablative margin were conducted blinded regarding the oncological outcome of the patient.

\section{Statistical analysis}

Based on previously published data by Nishikawa et al [28], we performed a sample size calculation in order to determine how many lesions had to be included in the present study to detect a possible difference in local tumor progression between ablations with sufficient $(>5 \mathrm{~mm})$ and insufficient minimal margins $(<5 \mathrm{~mm})$. Assuming a type 1 error of 0.05 and a type 2 error of 0.2 (thus yielding $80 \%$ statistical power), we found that a total of 77 lesions had to be analyzed to detect such a difference.

The distribution (parametric/non-parametric) of all variables was assessed using histograms. Baseline characteristics of the patients are expressed as mean \pm SD or median with interquartile range, as appropriate. Binary logistic regression (LR) was used to identify independent predictors of local tumor progression. In a second step, Cox regression modeling was utilized to visualize the hazard of LTP over time related to different covariates.

All statistical analyses were performed using SPSS Version 22 (SPSS Inc.). $P$ Values $<0.05$ were considered statistically significant.
Fig. 2 Diagram to illustrate the extension of MAM on a 12-h scale of an analogue watch with lesion (gray), ablation zone (dark gray), and extension of MAM (light blue)
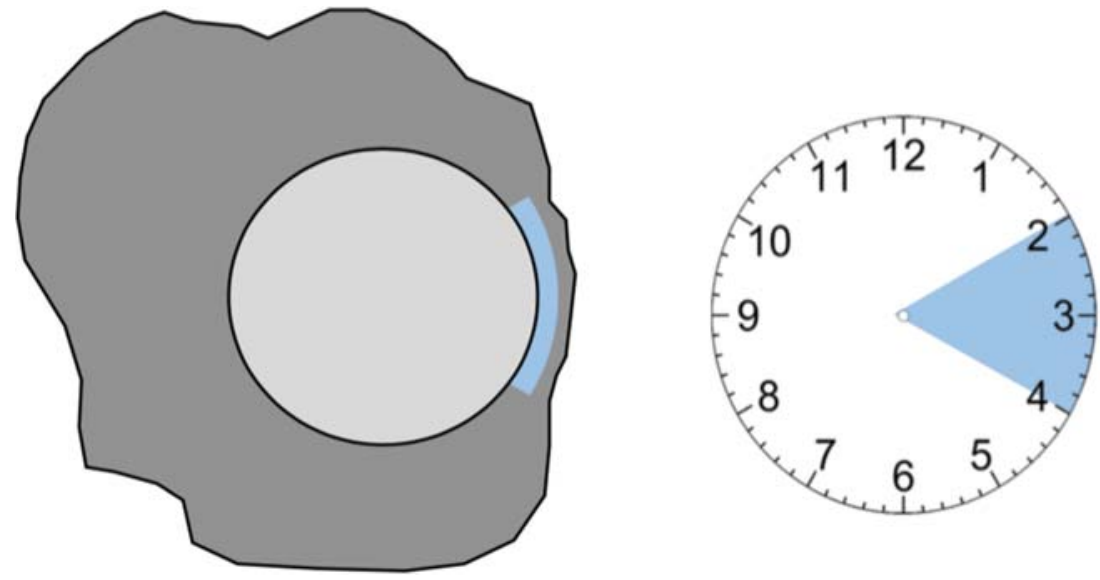
Fig. 3 A 63-year-old male patient treated with stereotactic RFA. a The arterial phase of pre-SRFA CT scan depicting a single HCC lesion (arrow) with a maximal diameter of $3.4 \mathrm{~cm}$ in liver segment II. b The arterial phase of post-SRFA CT scan with transient hyperemic rim around the ablation zone. $\mathbf{c}$ The after fusion of pre- and post-SRFA CT scan with manual registration by slight shifting (translation and rotation) in axial, coronal, and sagittal planes referring to intrahepatic structures such as vessel bifurcations. d The sagittal plane of CT image fusion with a MAM of $2.2 \mathrm{~mm}$ in clock position 5-6 $\mathrm{h}$ (arrow). e Local tumor progression after stereotactic RFA: contrast-enhanced CT scan 20.2 months after stereotactic RFA revealing a hypervascular, contrast-enhancing lesion in the arterial phase and with washout in delayed phase that is located immediately adjacent to the ablation zone
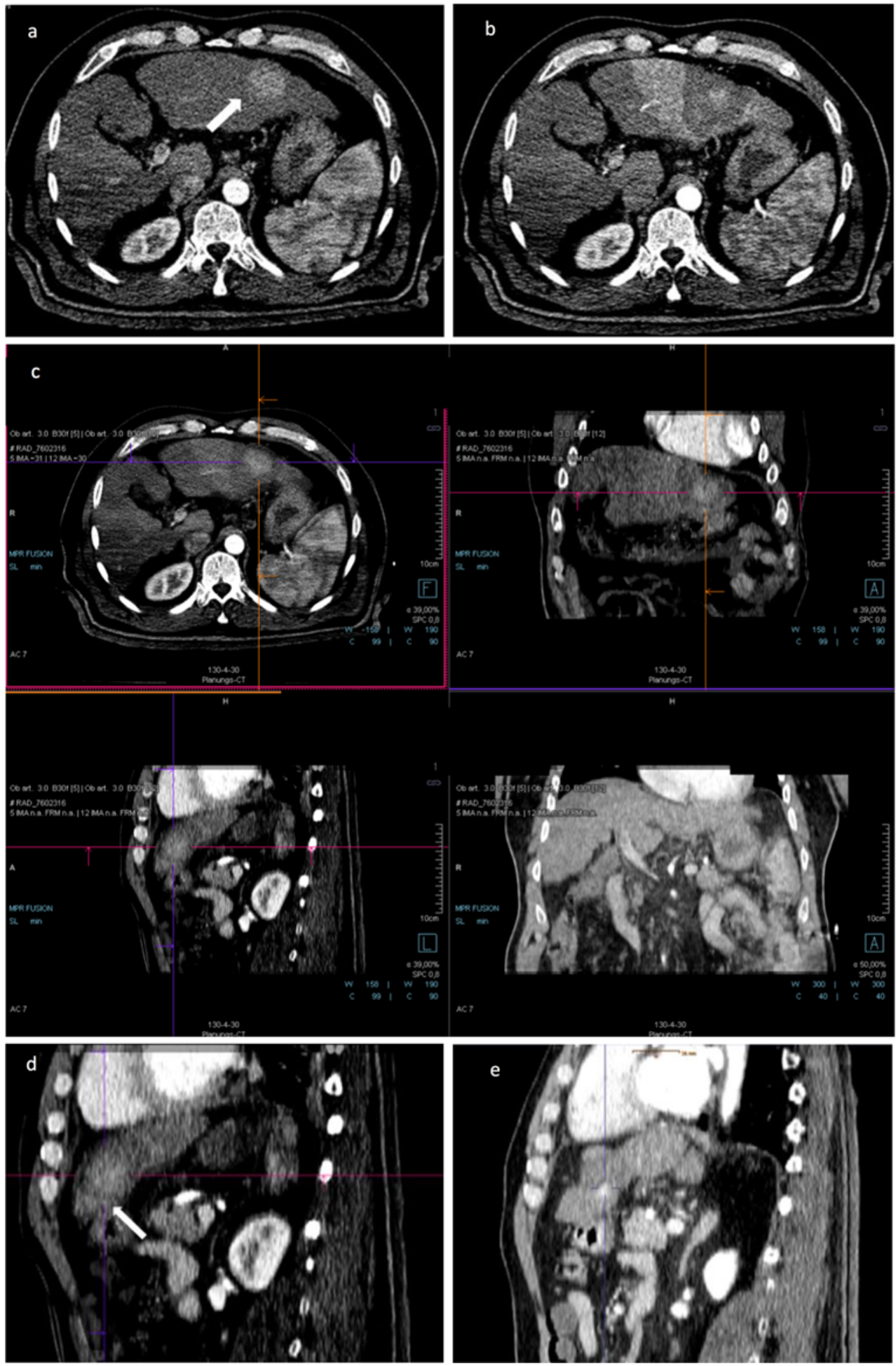

\section{Results}

Baseline characteristics of patients included in our analysis are shown in Table 1.

After exclusion of 97 lesions, 110 patients with $176 \mathrm{HCC}$ treated by stereotactic RFA were finally analyzed in this study (Fig. 4).
The mean overall follow-up period was $26.0 \pm 10.3$ months. The mean tumor size was $25.2 \pm 14.9 \mathrm{~mm}$. Seventy-seven lesions had no specificities regarding their location. The location of the remaining ninety-nine lesions was described as shown in Table 2. The median minimal ablative margin was $3(2-7) \mathrm{mm}$. Subdivided into groups, $110(62.5 \%)$ lesions had a MAM of less than $5 \mathrm{~mm}$, whereas sixty-six (37.5\%) lesions 
Fig. 4 An 88-year-old male patient treated with stereotactic RFA. a The arterial phase of preSRFA CT scan of a single HCC lesion with a maximal diameter of $6 \mathrm{~cm}$ in liver segments II, III, IVa, and IVb. b The late portal venous phase of post-SRFA CT scan showing clear demarcation of the ablation zone. $\mathbf{c}$ The after fusion of pre- and post-SRFA CT scan with manual registration by multiplanar slight shifting (translation and rotation) in axial, coronal, and sagittal planes referring to intrahepatic structures such as vessel bifurcation. d The axial plane of CT image fusion with a MAM of $2.5 \mathrm{~mm}$ in clock position 6-8 h (arrow). e MRI scan 24.9 months after ablation without evidence of LTP and progressively shrinking ablation zone
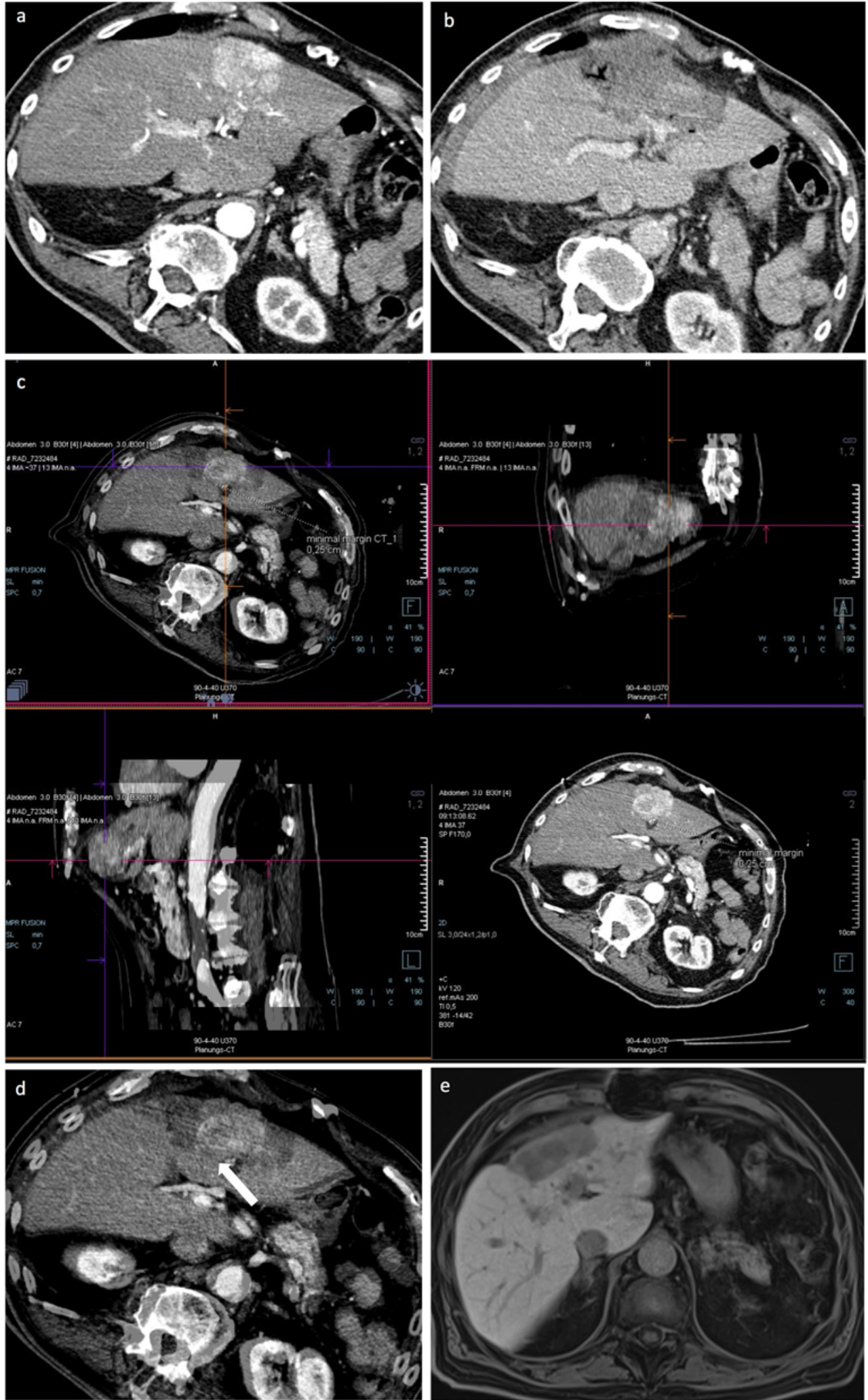

a MAM greater than $5 \mathrm{~mm}$. Overall LTP rate was 9 of 110 $(8.2 \%)$ on a patient level and 10 of $173(5.7 \%)$ per ablated HCC. Mean time to LTP was $16.9 \pm 9.3$ months. The median extension of the minimal margin was $2 \mathrm{~h}$ on a 12-h scale of an analogue watch in the corresponding plane (axial, coronal, or sagittal). In 96 (54.5\%) cases, a late arterial phase was utilized as a post-ablation image for image fusion, while in the remaining cases, a late portal venous phase had to be used. 
Fig. 5 Registration failure of a lesion in a 58-year-old male. a The arterial phase of pre-SRFA CT scan showing lesions in liver segment III with $4 \mathrm{~cm}$ in diameter (white arrow), in liver segment $\mathrm{V}$ with $3.2 \mathrm{~cm}$ (black arrow), and in liver segment VI with $1.6 \mathrm{~cm}$ (red circle). b The late portal venous phase of post-SRFA CT scan with treated lesions in liver segments $\mathrm{V}$ and VI. The lesion in liver segment $\mathrm{V}$ was treated in a second session 2 months later. c The successful fusion of pre- and postSRFA CT scan with the successful fusion of the lesion in liver segment V (MAM $2.6 \mathrm{~mm}$ in clock position 12-2 h). An adequate fusion for the lesion in liver segment VI was not possible due to its subcapsular location and extensive liver deformation
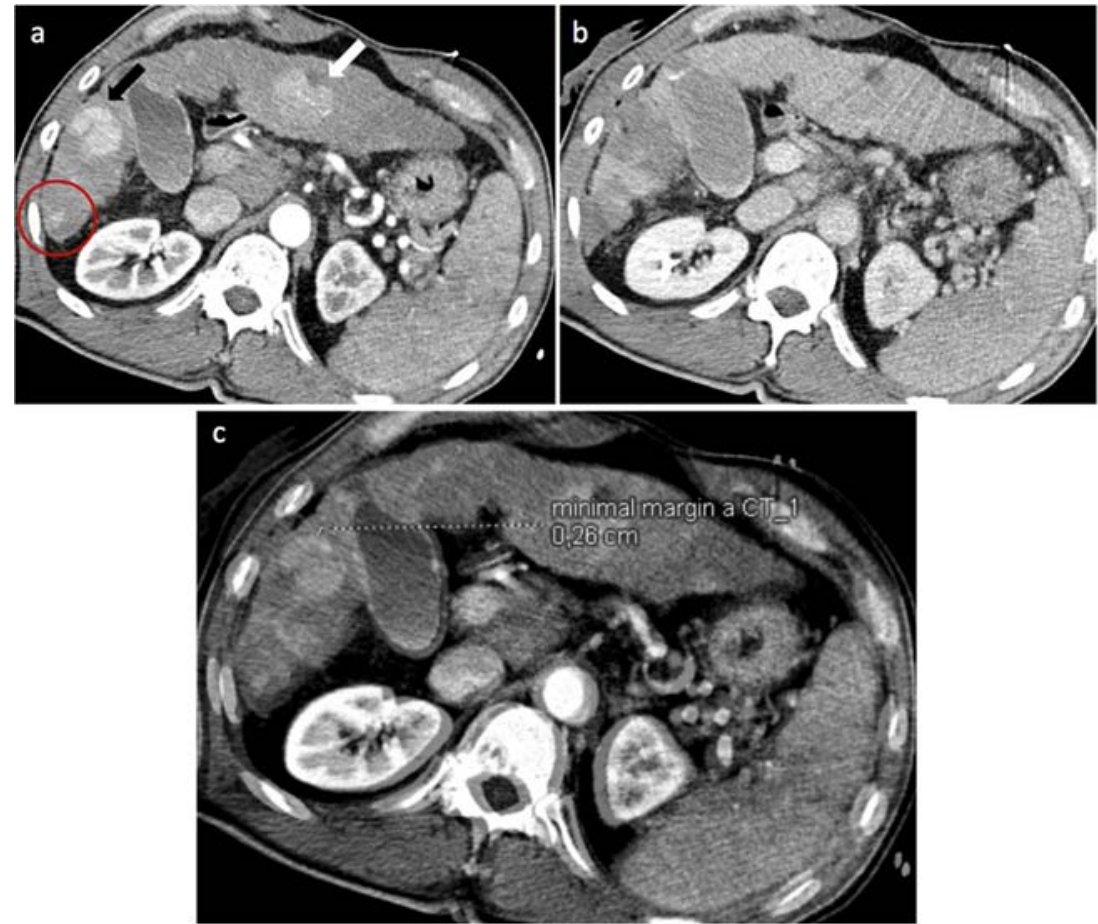

\section{Predictors of local tumor progression}

Our analysis revealed MAM as the only significant independent predictor of LTP $(p=0.036)$. The $R^{2}$ (Nagelkerke) of the model was calculated with 0.17 , which translates into a strong effect size (0.45) according to Cohen. For each millimeter increase of the MAM, a $30 \%$ reduction of the relative risk for LTP was found $(\mathrm{OR}=0.7,95 \%$ CI $0.5-0.98, p=0.036)$.

Table 1 Characteristics of 110 patients with a total of 176 HCCs ablated with stereotactic RFA. ${ }^{\dagger}$ Mean \pm standard deviation; ${ }^{\ddagger}$ median (interquartile range); $H B V$, hepatitis $\mathrm{B}$ virus; $H C V$, hepatitis $\mathrm{C}$ virus; $A F P, \alpha$-fetoprotein

\begin{tabular}{lc}
\hline Characteristics & Value \\
\hline Age (years) ${ }^{\dagger}$ & $63.7 \pm 10.2$ \\
Gender, $n(\%)$ & \\
Female & $20(18.2)$ \\
Male & $90(81.8)$ \\
Cirrhosis, $n(\%)$ & $95(86.4)$ \\
HBV & $6(5.5)$ \\
HCV & $19(17.3)$ \\
Fatty liver disease (AFLD and NAFLD) & $45(40.9)$ \\
Other & $25(27.7)$ \\
Child Pugh, $n(\%)$ & \\
Child A & $78(82.1)$ \\
Child B & $15(15.8)$ \\
Child C & $2(2.1)$ \\
AFP (ng/l) & $7(3.5-24.6)$ \\
No. of tumors treated per patient & $2(1-2)$ \\
\hline
\end{tabular}

No other variable such as tumor size or tumor location showed a significant influence on LTP.

The highly significant model of the binary logistic regression with the three possible determinants of LTP is shown in Table 3 .

To visualize the probability of LTP over time, Cox regression was utilized. As the MAM was found to be the only predictor of LTP in binary logistic regression, separate lines were coded for lesions with a MAM $>5 \mathrm{~mm}$ and $<5 \mathrm{~mm}$, respectively. No LTP was detected in lesions with a MAM $>5 \mathrm{~mm}$. The results are shown in Fig. 6 .

\section{Discussion}

Our data confirm that an ablation with a MAM $>5 \mathrm{~mm}$ can be considered successful, due to an extremely low probability of LTP, as already shown in other studies [24-26, 28, 29].

In our analysis, the median MAM was $3 \mathrm{~mm}$ and $37.5 \%$ (66/176) of the lesions were circumscribed by a MAM $>5 \mathrm{~mm}$. This indicates the enormous efficacy of stereotactic RFA, since in a similar retrospective study with image fusion, a circumscribed MAM $>5 \mathrm{~mm}$ after RFA could only be achieved in $2.7 \%$ (3/110) of all cases [30].

Our study shows that with image fusion and evaluation of the MAM, the risk of LTP can be significantly reduced, if not avoided altogether, once a circumscribed MAM $>5 \mathrm{~mm}$ has been achieved. Under those conditions, we did not observe any case of LTP, which underlines the crucial impact of the MAM on LTP.

In this context, it is important to underline the clinical need to fuse pre- and post-ablation images and determine the MAM 
Table 2 Characteristics of 176 HCCs ablated with stereotactic RFA. ${ }^{\dagger}$ Mean \pm standard deviation; ${ }^{\ddagger}$ median (interquartile range); MAM, minimal ablative margin; $L T P$, local tumor progression

\begin{tabular}{lc}
\hline Characteristics & Value \\
\hline Tumor size ${ }^{\dagger}$ [range] (mm) & $25.2 \pm 14.9[2-83]$ \\
Tumor size group, $n(\%)$ & \\
$<3 \mathrm{~cm}$ & $121(68.8)$ \\
$3-5 \mathrm{~cm}$ & $43(24.4)$ \\
$>5 \mathrm{~cm}$ & $12(6.8)$ \\
Tumor location & \\
Proximity to gallbladder & $4(2.3)$ \\
Proximity to major vessel & $32(18.2)$ \\
Proximity to extrahepatic organ & $11(6.3)$ \\
Subcapsular & $38(21.6)$ \\
Subphrenic & $14(8.0)$ \\
No specificities & $77(43.8)$ \\
Follow-up in months ${ }^{\dagger}$ & $26.0 \pm 10.3$ \\
MAM ${ }^{\ddagger}$ (mm) & $3(2-7)$ \\
MAM, $n$ (\%) & \\
$<5$ mm & $110(62.5)$ \\
$>5$ mm & $66(37.5)$ \\
MAM extension in hours ${ }^{\ddagger}$ & $2(1-2)$ \\
LTP, $n(\%)$ & $10(5.7)$ \\
Time to LTP in months ${ }^{\dagger}$ & $16.9 \pm 9.3$ \\
\hline
\end{tabular}

immediately after the ablation, such that an ablation can be repeated or continued in locations where a MAM $>5 \mathrm{~mm}$ could not be guaranteed.

In previous studies, the MAM was assessed through sideby-side juxtaposition of pre- and post-ablation CT scans with automatic rigid registration software and final manual adjustment [31, 32]; a similar approach is used in our daily clinical practice. This includes automatic mutual information based rigid registration and, in addition, definition of corresponding anatomical landmarks on the different image datasets [12]. However, this method is rather challenging even for very experienced radiologists, considering the need to determine the MAM in three planes in a side-by-side manner. As a result, it contains many potential sources of error.

Table 3 Binary logistic regression. Model with three possible determinants of LTP. MAM, minimal ablative margin; $C I$, confidence interval; $L T P$, local tumor progression

\begin{tabular}{lllll}
\hline & Odds ratio & $95 \% \mathrm{CI}$ & $p$ value & $R^{2}$ Nagelkerke \\
\hline Tumor size & 1.023 & $0.987-1.060$ & 0.222 & \\
MAM & 0.700 & $0.502-0.977$ & 0.036 & \\
Tumor location & 0.937 & $0.663-1.324$ & 0.714 & \\
Model summary & & & 0.012 & 0.171 \\
\hline
\end{tabular}

Our study shows that an image fusion using commercially available rigid imaging registration software is possible and the assessment of the MAM with it can be used as an intraoperative tool to evaluate the treatment efficacy of stereotactic RFA. Nevertheless, a limitation has to be discussed. An appropriate image fusion is considerably complex. Despite getting used to the software and the procedure, a fusion with a lesion demarking clearly from the surrounding liver parenchyma and near intrahepatic landmarks, including the quantitative evaluation of the minimal margin, could not be performed in under $15 \mathrm{~min}$. Therefore, improved (semi-)automatic software is desperately needed, with which an image fusion of pre- and postablation CT scans is feasible in only a few minutes and hence applicable in daily clinical practice. An alternative image fusion software platform for volumetric assessment of ablation completeness was recently used in a retrospective study and may be a promising candidate [33]. Still, further investigations are needed before introducing it into daily clinical practice.

Regarding the issue of unsuccessful registration due to extensive liver deformation that encountered in registration of very large or multiple tumors, the implementation of non-rigid registration algorithms may be beneficial. However, further investigation in this field is required.

Unlike previous findings [26, 28, 34], our data revealed the MAM as the only significant independent predictor for LTP. Tumor size, often described as a determinant of LTP in patients treated with RFA, had no significant influence on LTP in our study. This is in line with previous studies about stereotactic RFA, where tumor size did not have any influence on LTP [14, $16,17,19]$. This can be explained by multiple probe positioning with overlapping ablation zones in stereotactic RFA and the 3D navigated stereotactic planning, which is a crucial distinction to conventional CT- or US-guided ablation. This is a very important finding, given that the AASLD currently considers RFA as an equal treatment to surgical resection only in single tumors $<2.5 \mathrm{~cm}$ [5]. Indeed, the mean tumor size in our study was $25.2 \pm 14.9 \mathrm{~mm}$ and thus, many lesions did not fit the abovementioned criteria. Using stereotactic RFA, it is possible to achieve a curative treatment of multiple lesions and lesions up to $10 \mathrm{~cm}$ in diameter [12]. Therefore, from the oncological point of view, stereotactic RFA challenges surgical resection as first line treatment, even in patients with lesions $>2.5 \mathrm{~cm}$.

Tumor location also had no significant influence on LTP. This was surprising, considering important location-related influence factors, like the heat sink effect caused by blood flow at the site of ablation. It is known that this effect can be overcome by increased duration and power of ablation, with ablation probes being preferentially positioned next to the vessel site. In conventional RFA, such accurate probe positioning is not always possible and very challenging due to respiratory motion of the patient; it therefore carries a higher risk for procedure-related complications. As mentioned above, SRFA is performed under general anesthesia with full 
Fig. 6 Cox regression with hazard function of LTP over time, subdivided in MAM $<5 \mathrm{~mm}$ (blue) and MAM $>5 \mathrm{~mm}$ (black)

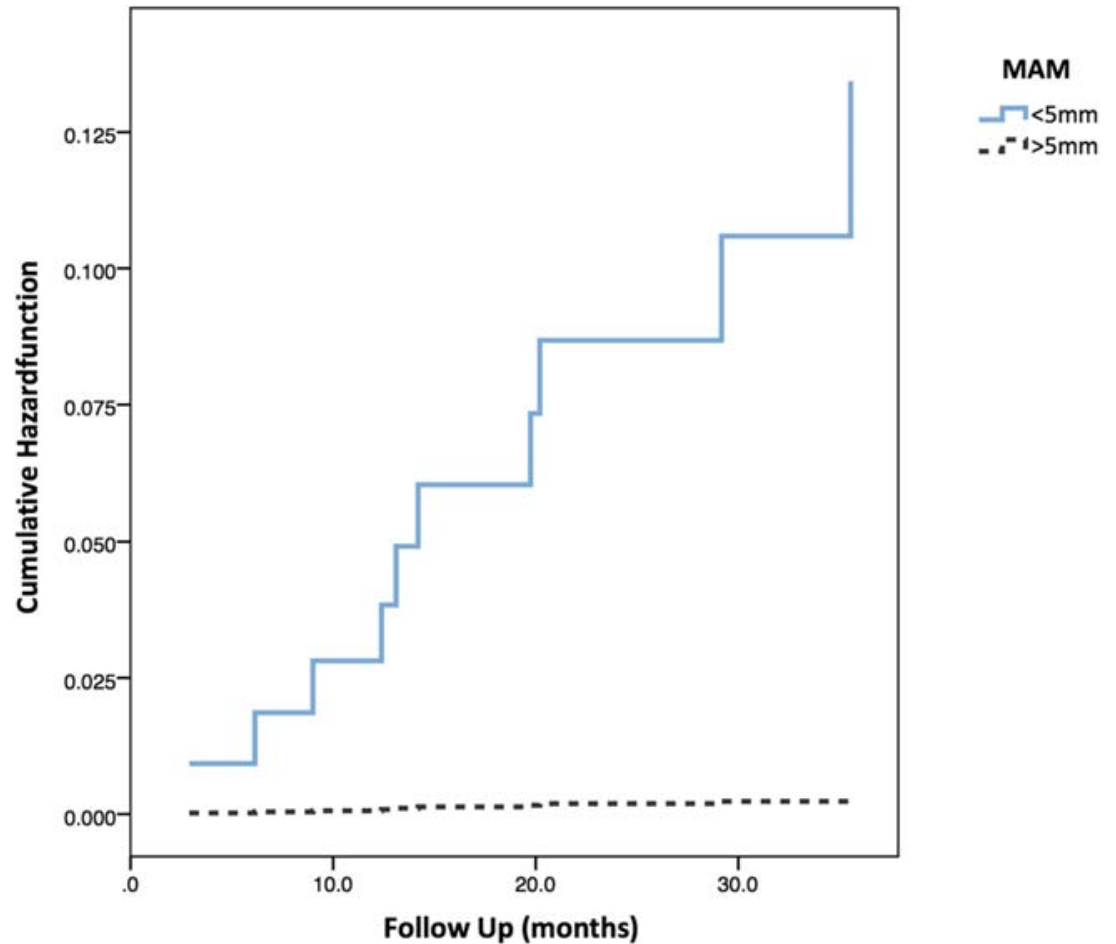

relaxation, and the probes are positioned in tracheal tube disconnection. This guarantees an exact probe positioning as previously planned and avoids imprecisions due to respiratory motion. Therefore, an ablation is possible even in difficult to reach locations. Clearly, lesions closer than $1 \mathrm{~cm}$ to the central biliary structures are a priori not feasible for stereotactic RFA.

Overall, our data confirm the enormous efficacy of stereotactic RFA in selected patients with HCC considering the very low LTP rate of only $5.7 \%$. This finding is in line with a recently published study [15], where the efficacy of stereotactic RFA was evaluated by histopathological examination after bridging therapy for liver transplantation. Complete pathological response in the explanted liver specimen was achieved in 183 of 188 nodules (97.3\%), and in 50 of 52 nodules $>3 \mathrm{~cm}$ (96.2\%). For these reasons, the treatment plan of every patient should be established in a multidisciplinary tumor board with hepatologists, oncologists, transplant surgeons, and interventional radiologists, considering stereotactic RFA a valid curative option [35].

We are well aware of the limitations of our study, as it is a single-center study with retrospective character. Nevertheless, the results are clear and encouraging, justifying specialized training in stereotactic techniques as well as additional costs related to infrastructure.

In summary our study revealed three important findings.

First, an immediate assessment of the minimal ablative margin (MAM) can be used as an intraoperative tool to evaluate the treatment success in patients treated with stereotactic RFA as it appears to be the only significant and independent predictor of LTP.

Second, a MAM > $5 \mathrm{~mm}$ has to be achieved to avoid local tumor progression (LTP) and to consider an ablation as successful.
Third, image fusion using commercially available rigid imaging registration software is possible, even though considerably complex. Therefore, improved (semi-)automatic software is desperately needed.

Funding information Open access funding provided by University of Innsbruck and Medical University of Innsbruck. The authors state that this work has not received any funding.

\section{Compliance with ethical standards}

Guarantor The scientific guarantor of this publication is Reto Bale.

Conflict of interest Reto Bale is a consultant of CASCINATION. Amilcar Alzaga is an employee of Siemens Healthineers.

The other authors do not have any conflicts of interest to disclose.

Statistics and biometry One of the authors has significant statistical expertise.

No complex statistical methods were necessary for this paper.

Informed consent Written informed consent was waived by the Institutional Review Board.

Ethical approval Institutional Review Board approval was obtained.

Methodology

- Retrospective

- Observational

- Performed at one institution

Open Access This article is licensed under a Creative Commons Attribution 4.0 International License, which permits use, sharing, adaptation, distribution and reproduction in any medium or format, as long as 
you give appropriate credit to the original author(s) and the source, provide a link to the Creative Commons licence, and indicate if changes were made. The images or other third party material in this article are included in the article's Creative Commons licence, unless indicated otherwise in a credit line to the material. If material is not included in the article's Creative Commons licence and your intended use is not permitted by statutory regulation or exceeds the permitted use, you will need to obtain permission directly from the copyright holder. To view a copy of this licence, visit http://creativecommons.org/licenses/by/4.0/.

\section{References}

1. Forner A, Llovet JM, Bruix J (2012) Hepatocellular carcinoma. Lancet 379:1245-1255

2. El-Serag HB (2011) Hepatocellular carcinoma. N Engl J Med 365: $1118-1127$

3. Facciorusso A, Di Maso M, Muscatiello N (2016) Microwave ablation versus radiofrequency ablation for the treatment of hepatocellular carcinoma: a systematic review and meta-analysis. Int $\mathrm{J}$ Hyperthermia 32:339-344

4. Langenbach MC (2019) RFA vs resection of HCC: exploring the past to improve the future. Eur Radiol 29:2677-2678

5. Heimbach JK, Kulik LM, Finn RS et al (2018) AASLD guidelines for the treatment of hepatocellular carcinoma. Hepatology 67:358-380

6. Kasugai H, Osaki Y, Oka H, Kudo M, Seki T, Osaka Liver Cancer Study Group (2007) Severe complications of radiofrequency ablation therapy for hepatocellular carcinoma: an analysis of 3,891 ablations in 2,614 patients. Oncology 72(Suppl 1):72-75

7. Livraghi T, Meloni F, Di Stasi M et al (2008) Sustained complete response and complications rates after radiofrequency ablation of very early hepatocellular carcinoma in cirrhosis: is resection still the treatment of choice? Hepatology 47:82-89

8. N'Kontchou G, Mahamoudi A, Aout M et al (2009) Radiofrequency ablation of hepatocellular carcinoma: long-term results and prognostic factors in 235 Western patients with cirrhosis. Hepatology 50:1475-1483

9. Livraghi T, Solbiati L, Meloni MF, Gazelle GS, Halpern EF, Goldberg SN (2003) Treatment of focal liver tumors with percutaneous radio-frequency ablation: complications encountered in a multicenter study. Radiology 226:441-451

10. Uhlig J, Sellers CM, Stein SM, Kim HS (2019) Radiofrequency ablation versus surgical resection of hepatocellular carcinoma: contemporary treatment trends and outcomes from the United States National Cancer Database. Eur Radiol 29:2679-2689

11. Cha DI, Kang TW, Song KD et al (2019) Radiofrequency ablation for subcardiac hepatocellular carcinoma: therapeutic outcomes and risk factors for technical failure. Eur Radiol 29:2706-2715

12. Bale R, Widmann G, Haidu M (2011) Stereotactic radiofrequency ablation. Cardiovasc Intervent Radiol 34:852-856

13. Bale R, Widmann G, Stoffner DI (2010) Stereotaxy: breaking the limits of current radiofrequency ablation techniques. Eur J Radiol 75:32-36

14. Haidu M, Dobrozemsky G, Schullian P et al (2012) Stereotactic radiofrequency ablation of unresectable intrahepatic cholangiocarcinomas: a retrospective study. Cardiovasc Intervent Radiol 35:1074-1082

15. Bale R, Schullian P, Eberle G et al (2019) Stereotactic radiofrequency ablation of hepatocellular carcinoma: a histopathological study in explanted livers. Hepatology 70(3):840-850

16. Bale R, Schullian P, Schmuth M, Widmann G, Jaschke W, Weinlich G (2016) Stereotactic radiofrequency ablation for metastatic melanoma to the liver. Cardiovasc Intervent Radiol 39:1128-1135

17. Bale R, Richter M, Dünser M, Levy E, Buchberger W, Schullian P (2018) Stereotactic radiofrequency ablation for breast cancer liver metastases. J Vasc Interv Radiol 29:262-267
18. Bale R, Freund M, Bodner G, Kovacs P, Jaschke W (2002) Precise computer-assisted liver tumor puncture for biopsy and thermal ablation. 88th Scientific Assembly and Annual Meeting of the Radiological-Society-of-North-AmericaRadiology 225(S):242

19. Bale R, Widmann G, Schullian P et al (2012) Percutaneous stereotactic radiofrequency ablation of colorectal liver metastases. Eur Radiol 22:930-937

20. Bale R, Widmann G (2007) Navigated CT-guided interventions. Minim Invasive Ther Allied Technol 16:196-204

21. Lafaro K, Grandhi MS, Herman JM, Pawlik TM (2016) The importance of surgical margins in primary malignancies of the liver. $\mathrm{J}$ Surg Oncol 113:296-303

22. Dong S, Wang Z, Wu L, Qu Z (2016) Effect of surgical margin in R0 hepatectomy on recurrence-free survival of patients with solitary hepatocellular carcinomas without macroscopic vascular invasion. Medicine (Baltimore) 95:e5251

23. Shi M, Guo RP, Lin XJ et al (2007) Partial hepatectomy with wide versus narrow resection margin for solitary hepatocellular carcinoma: a prospective randomized trial. Ann Surg 245:36-43

24. Motoyama T, Ogasawara S, Chiba T et al (2014) Coronal reformatted CT images contribute to the precise evaluation of the radiofrequency ablative margin for hepatocellular carcinoma. Abdom Imaging 39:262-268

25. Nakazawa T, Kokubu S, Shibuya A et al (2007) Radiofrequency ablation of hepatocellular carcinoma: correlation between local tumor progression after ablation and ablative margin. AJR Am J Roentgenol 188:480-488

26. Hocquelet A, Trillaud H, Frulio N et al (2016) Three-dimensional measurement of hepatocellular carcinoma ablation zones and margins for predicting local tumor progression. J Vasc Interv Radiol 27:1038-45.e2

27. European Association for the Study of the Liver (2018) EASL clinical practice guidelines: management of hepatocellular carcinoma. J Hepatol 69:182-236

28. Nishikawa $H$, Inuzuka $T$, Takeda $H$ et al (2011) Percutaneous radiofrequency ablation therapy for hepatocellular carcinoma: a proposed new grading system for the ablative margin and prediction of local tumor progression and its validation. J Gastroenterol 46:1418-1426

29. Jiang C, Liu B, Chen S, Peng Z, Xie X, Kuang M (2018) Safety margin after radiofrequency ablation of hepatocellular carcinoma: precise assessment with a three-dimensional reconstruction technique using CT imaging. Int J Hyperthermia 34:1135-1141

30. Kim YS, Lee WJ, Rhim H, Lim HK, Choi D, Lee JY (2010) The minimal ablative margin of radiofrequency ablation of hepatocellular carcinoma ( $>2$ and $<5 \mathrm{~cm}$ ) needed to prevent local tumor progression: 3D quantitative assessment using CT image fusion. AJR Am J Roentgenol 195:758-765

31. Tomonari A, Tsuji K, Yamazaki H et al (2013) Feasibility of fused imaging for the evaluation of radiofrequency ablative margin for hepatocellular carcinoma. Hepatol Res 43:728-734

32. Makino Y, Imai Y, Igura T et al (2013) Utility of computed tomography fusion imaging for the evaluation of the ablative margin of radiofrequency ablation for hepatocellular carcinoma and the correlation to local tumor progression. Hepatol Res 43:950-958

33. Solbiati M, Muglia R, Goldberg SN et al (2019) A novel software platform for volumetric assessment of ablation completeness. Int J Hyperthermia 36:337-343

34. Nishikawa H, Osaki Y, Iguchi E et al (2013) Radiofrequency ablation for hepatocellular carcinoma: the relationship between a new grading system for the ablative margin and clinical outcomes. J Gastroenterol 48:951-965

35. Putzer D, Schullian P, Braunwarth E et al (2018) Integrating interventional oncology in the treatment of liver tumors. Eur Surg 50:117-124

Publisher's note Springer Nature remains neutral with regard to jurisdictional claims in published maps and institutional affiliations. 